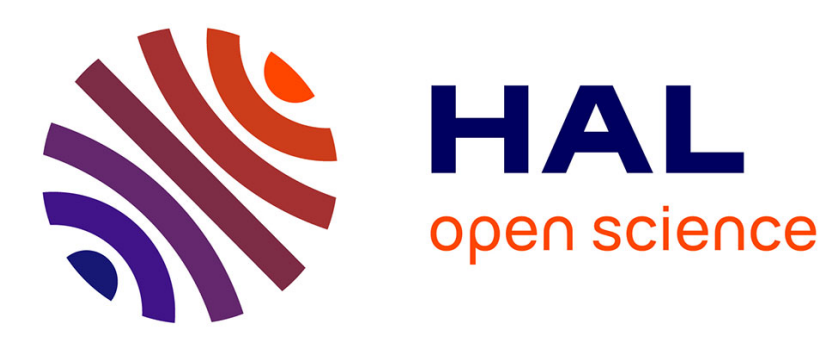

\title{
Theory of red blood cell oscillations in an ultrasound field
}

\author{
Kristoffer Johansen, Eitan Kimmel, Michiel Postema
}

\section{To cite this version:}

Kristoffer Johansen, Eitan Kimmel, Michiel Postema. Theory of red blood cell oscillations in an ultrasound field. Archives of Acoustics, 2017, 42 (1), pp.121-126. 10.1515/aoa-2017-0013 . hal03192804

\section{HAL Id: hal-03192804 \\ https://hal.science/hal-03192804}

Submitted on 13 Apr 2021

HAL is a multi-disciplinary open access archive for the deposit and dissemination of scientific research documents, whether they are published or not. The documents may come from teaching and research institutions in France or abroad, or from public or private research centers.
L'archive ouverte pluridisciplinaire HAL, est destinée au dépôt et à la diffusion de documents scientifiques de niveau recherche, publiés ou non, émanant des établissements d'enseignement et de recherche français ou étrangers, des laboratoires publics ou privés.

\section{다(1)(2)}

Distributed under a Creative Commons Attribution - ShareAlikel 4.0 International 


\title{
Theory of Red Blood Cell Oscillations in an Ultrasound Field
}

\author{
Kristoffer JOHANSEN ${ }^{(1)}$, Eitan $\operatorname{KIMMEL}^{(2)}$, Michiel POSTEMA ${ }^{(3),(4)}$ \\ (1) School of Engineering, James Watt Building \\ University of Glasgow \\ Glasgow, G12 8QQ, Scotland; e-mail: k.johansen.1@research.gla.ac.uk \\ ${ }^{(2)}$ Faculty of Biomedical Engineering, Technion - Israel Institute of Technology \\ Haifa 32000, Israel; e-mail: eitan@bm.technion.ac.il \\ ${ }^{(3)}$ Institute of Fundamental Technological Research \\ Polish Academy of Sciences \\ Pawińskiego 5B, 02-106 Warsaw, Poland; e-mail: mpostema@ippt.pan.pl \\ (4) School of Electrical and Information Engineering, Chamber of Mines Building \\ University of the Witwatersrand \\ 1 Jan Smuts Avenue, Braamfontein, Johannesburg 2050, South Africa
}

(received June 3, 2016; accepted November 30, 2016)

\begin{abstract}
Manipulating particles in the blood pool with noninvasive methods has been of great interest in therapeutic delivery. Recently, it was demonstrated experimentally that red blood cells can be forced to translate and accumulate in an ultrasound field. This acoustic response of the red blood cells has been attributed to sonophores, gas pockets that are formed under the influence of a sound field in the innermembrane leaflets of biological cells. In this paper, we propose a simpler model: that of the compressible membrane. We derive the spatio-temporal cell dynamics for a spherically symmetric single cell, whilst regarding the cell bilayer membrane as two monolayer Newtonian viscous liquids, separated by a thin gas void.

When applying the newly-derived equations to a red blood cell, it is observed that the void inside the bilayer expands to multiples of its original thickness, even at clinically safe acoustic pressure amplitudes. For causing permanent cell rupture during expansion, however, the acoustic pressure amplitudes needed would have to surpass the inertial cavitation threshold by a factor 10 .

Given the incompressibility of the inner monolayer, the radial oscillations of a cell are governed by the same set of equations as those of a forced antibubble. Evidently, these equations must hold for liposomes under sonication, as well.
\end{abstract}

Keywords: spatio-temporal cell dynamics; Rayleigh-Plesset equation; spherical cell; red blood cell; erythrocyte; sonophore.

\section{Introduction}

Manipulating particles in the blood pool with noninvasive methods has been of great interest in therapeutic delivery, about which a review has been published by Delalande et al. (2012). Ultrasonic equipment is commonly used for noninvasive manipulation, owing to its reliability, safety, availability, and low operating cost. Following numerous nondestructive and destructive experimental studies on microbubbles under sonication near biological cells, e.g., PREnTICE et al. (2005), VAN WAMEL et al. (2006), KUDO et al.
(2009), and Delalande et al. (2011), it was speculated that cells themselves respond to sound by oscillating (KRASOVITSKI et al., 2011), which might lead to novel ways of noninvasive cell manipulation, filtration, and even eradication (Walther, Postema, 2016).

Recently, MAZZAWI et al. (2015) demonstrated experimentally that red blood cells can be forced to translate and accumulate in an ultrasound field. This acoustic response of the red blood cells has been attributed to sonophores, gas pockets that are formed under the influence of a sound field in the innermembrane leaflets of biological cells (KRASOVITSKI 
et al., 2011). Under sonication, such trapped gas pockets must oscillate in a similar way as encapsulated gas microbubbles do.

Although the existence of sonophores would explain experimentally observed phenomena, such as the attraction of oscillating microbubbles to fixated cells (Delalande et al., 2011) and the occurrence of the transient formation of pores in cell membranes in the absence of microbubbles (BAO et al., 1997), there might be a more straightforward explanation for the behaviour observed.

In this paper, we propose a simpler model: that of the compressible membrane. Initially, we follow the derivation for antibubble dynamics of KOTOPOULIS et al. (2015) step by step whilst replacing only few parameters, but from Subsec. 2.4 we deviate by including an outer cell membrane, following the derivation of encapsulated bubbles dynamics by Church (1995).

\section{Theory}

Let us consider a spherical cell, as schematically represented in Fig. 1. We regard the inner cell structure as an incompressible liquid of radius $R_{1}$. Instead of regarding the surrounding bilayer membrane as one layer, we split it up into three components (BOAL, 2012): an inner monolayer Newtonian viscous liquid membrane of inner radius $R_{1}$ and outer radius $R_{2}$, an outer monolayer Newtonian viscous liquid membrane of inner radius $R_{3}$ and outer radius $R_{4}$, and a gas void separating both monolayers of inner radius $R_{2}$ and outer radius $R_{3}$. Even though actual cells are rarely spherical, we may disregard this fact, as the radial dynamics are predominantly determined by the surface pressure of the surface with the greatest curvature (ISENBERG, 1992).

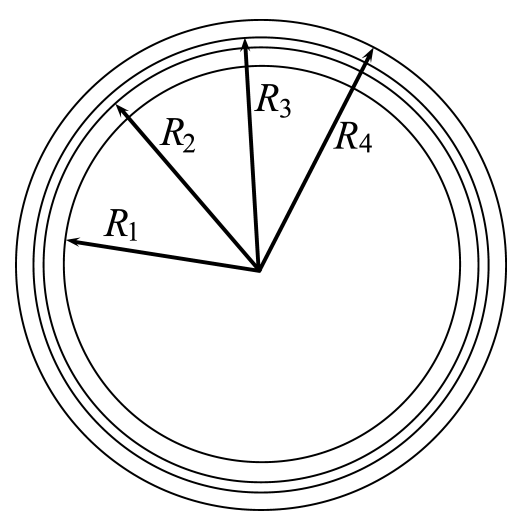

Fig. 1. Schematic representation of a spherical cell consisting of a liquid core of radius $R_{1}$, surrounded by a monolayer Newtonian viscous liquid membrane of outer radius $R_{2}$, a compressible gas void of outer radius $R_{3}$, and a monolayer Newtonian viscous liquid membrane of outer radius $R_{4}$.
Because the monolayer membranes are considered Newtonian viscous liquids, they are incompressible, as is the intracellular liquid. Consequently, the inner membrane radii do not respond to pressure changes. However, the gas void separating the inside and outside membranes must abide by the same thermodynamics that cavitation bubbles do when subjected to a sound field.

\subsection{Fundamental equation of cell dynamics}

Let us consider a polytropic gas void, surrounding the spherical incompressible liquid core of radius $R_{2}$ as presented in Fig. 1. For now, we ignore the outer monolayer membrane.

We are assuming adiabatic conditions. Furthermore, no mass exchange between the respective interfaces is assumed.

Following the derivation for antibubble dynamics of Kotopoulis et al. (2015) and introducing a driving function $P(t)$, the fundamental equation of cell dynamics becomes:

$$
\begin{aligned}
& R_{3} \ddot{R}_{3}+\frac{3}{2} \dot{R}_{3}^{2} \\
& =\frac{1}{\rho_{\mathrm{L}}}\left[\left(p_{0}+\frac{2 \sigma}{R_{30}}\right)\left(\frac{R_{30}^{3}-R_{2}{ }^{3}}{R_{3}{ }^{3}-R_{2}{ }^{3}}\right)^{\gamma}-\frac{2 \sigma}{R_{3}}-p_{0}-P(t)\right],
\end{aligned}
$$

where $p_{0}$ is the ambient pressure, $R_{30}$ is the initial outer radius of the gas void, $\gamma$ is the polytropic exponent of the gas inside the void, $\rho_{\mathrm{L}}$ is the density of the liquid surrounding the cell, and $\sigma$ is the surface tension.

\subsection{Cell dynamics in a Newtonian viscous fluid}

The viscosity $\eta_{\mathrm{L}}$ of a Newtonian viscous fluid is by definition equal to the rate of strain $\Delta \varepsilon / \Delta t$.

Again, following the derivation for antibubble dynamics of KотороULIS et al. (2015) and introducing a driving function $P(t)$, the fundamental equation of cell dynamics in a Newtonian viscous fluid becomes:

$$
\begin{aligned}
R_{3} \ddot{R}_{3}+\frac{3}{2} \dot{R}_{3}^{2}= & \frac{1}{\rho_{\mathrm{L}}}\left[\left(p_{0}+\frac{2 \sigma}{R_{30}}\right)\left(\frac{R_{30}^{3}-R_{2}{ }^{3}}{R_{3}{ }^{3}-R_{2}{ }^{3}}\right)^{\gamma}\right. \\
& \left.-\frac{2 \sigma}{R_{3}}-\frac{4 \eta_{\mathrm{L}}}{R_{3}} \dot{R}_{3}-p_{0}-P(t)\right] .
\end{aligned}
$$

This is the Rayleigh-Plesset-like equation for a cell in a Newtonian viscous fluid, which can only be applied if the surrounding fluid is incompressible and the gas is polytropic. The equation is a second-order nonlinear ordinary differential equation.

\subsection{Linear analysis for an unrestrained cell}

Let us assume that (2) has a solution of the form

$$
R_{3}(t)=R_{30}[1+\xi(t)]
$$


for small excursions $\xi$ of the outer surface of the void, i.e., $\xi \ll R_{30}$. Then, (2) can be linearised and represented by a mass-spring-dashpot system (PostemA, 2011).

The linear natural angular resonance frequency $\omega_{0}$ of an unrestrained cell is given by:

$$
\omega_{0}^{2}=\frac{1}{R_{30}^{2} \rho_{\mathrm{L}}}\left[\frac{3 \gamma p_{\mathrm{g} 0}}{1-\left(\frac{R_{2}}{R_{30}}\right)^{3}}-\frac{2 \sigma}{R_{30}}\right],
$$

where $p_{\mathrm{g} 0}$ is the initial gas pressure inside the void.

It can be observed that the linear damped resonance frequency of a gas void is increased by the cubic ratio of the inner cell liquid radius and the initial radius compared to that of a regular gas bubble.

If a cell is suspended in a Newtonian viscous fluid, it resonates with a linear damped natural angular resonance frequency $\omega_{\mathrm{d}}$, given by (2.2.7) in KoTOPOULIS et al. (2015):

$$
\omega_{\mathrm{d}}^{2}=\frac{1}{R_{30}^{2} \rho_{\mathrm{L}}}\left[\frac{3 \gamma p_{\mathrm{g} 0}}{1-\left(\frac{R_{2}}{R_{30}}\right)^{3}}-\frac{2 \sigma}{R_{30}}-\frac{4 \eta_{\mathrm{L}}{ }^{2}}{R_{30}{ }^{2} \rho_{\mathrm{L}}}\right] .
$$

It can be observed from (5) that the linear damped resonance frequency decreases when the viscosity of the surrounding fluid increases.

\subsection{Presence of the outer cell membrane}

Now let us take into account that the gas void is surrounded by a restraining layer. Both the liquid composing the membrane and the outer surrounding liquid are assumed to be viscous and incompressible. Assuming no mass exchange between the respective interfaces, the radial velocity $v(r, t)$ in the membrane and in the surrounding fluid at a distance $r$ from the centre of the cell can be expressed as (LEIGHTON, 1994):

$$
v(r, t)=\frac{R_{3}^{2}}{r^{2}} \dot{R}_{3}
$$

If $R_{3}<r<R_{4}, v(r, t)$ denotes the velocity inside the membrane. If $r>R_{4}, v(r, t)$ denotes the radial velocity of the surrounding liquid. From the assumption of an incompressible membrane it can be shown that

$$
R_{4}{ }^{3}-R_{3}{ }^{3}=R_{40}{ }^{3}-R_{30}{ }^{3}
$$

and

$$
R_{3}^{2} \dot{R}_{3}=R_{4}^{2} \dot{R}_{4}
$$

where $R_{40}$ is the initial cell radius.

From conservation of radial momentum (LANDAU, Lifshitz, 1986), it follows that

$$
\rho_{\mathrm{M}}\left(\frac{\partial v}{\partial t}+v \frac{\partial v}{\partial r}\right)=-\frac{\partial p}{\partial r}+\frac{\partial \tau_{r r}^{\mathrm{M}}}{\partial r}+\frac{3 \tau_{r r}^{\mathrm{M}}}{r}
$$

and

$$
\rho_{\mathrm{L}}\left(\frac{\partial v}{\partial t}+v \frac{\partial v}{\partial r}\right)=-\frac{\partial p}{\partial r}+\frac{\partial \tau_{r r}^{\mathrm{L}}}{\partial r}+\frac{3 \tau_{r r}^{\mathrm{L}}}{r}
$$

where $\rho_{\mathrm{M}}$ is the the density of the membrane, $\rho_{\mathrm{L}}$ is the density of the surrounding liquid, $p$ is the pressure in the membrane or the liquid, $\tau_{r r}^{\mathrm{M}}$ is the viscous stress tensor in the membrane, and $\tau_{r r}^{\mathrm{L}}$ is the viscous stress in the liquid. Equation (9) is integrated from $R_{3}$ to $R_{4}$, and (10) is integrated from $R_{4}$ to infinity, substituting (6) for $v$. It is here assumed that the contribution to the radial momentum from the gas inside the void can be neglected. Combining these two integrals, the result can be expressed as

$$
\begin{aligned}
\rho_{\mathrm{L}} R_{3} \ddot{R}_{3}\left[1+\left(\frac{\rho_{\mathrm{L}}-\rho_{\mathrm{M}}}{\rho_{\mathrm{M}}}\right) \frac{R_{3}}{R_{4}}\right] \\
+\rho_{\mathrm{L}} \dot{R}_{3}^{2}\left[\frac{3}{2}+\left(\frac{\rho_{\mathrm{L}}-\rho_{\mathrm{M}}}{\rho_{\mathrm{M}}}\right)\left(\frac{4 R_{4}{ }^{3}-R_{3}{ }^{3}}{R_{4}{ }^{3}}\right)\right] \\
=P_{\mathrm{M}}\left(R_{3}, t\right)-P_{\mathrm{M}}\left(R_{4}, t\right)+P_{\mathrm{L}}\left(R_{4}, r\right) \\
+\tau_{r r}^{\mathrm{M}}\left(R_{4}, t\right)-\tau_{r r}^{\mathrm{M}}\left(R_{3}, t\right)-\tau_{r r}^{\mathrm{L}}\left(R_{4}, t\right) \\
+3 \int_{R_{3}}^{R_{4}} \frac{\tau_{r r}^{\mathrm{M}}}{r} \mathrm{~d} r+3 \int_{R_{4}}^{\infty} \frac{\tau_{r r}^{\mathrm{L}}}{r} \mathrm{~d} r,
\end{aligned}
$$

where $P_{\mathrm{M}}\left(R_{1}, t\right)$ and $P_{\mathrm{M}}\left(R_{2}, t\right)$ are the pressures in the membrane at the inner and outer interfaces, respectively, $P_{\mathrm{L}}\left(R_{2}, t\right)$ is the pressure in the liquid at the outer interface, $\tau_{r r}^{\mathrm{M}}\left(R_{1}, t\right)$ and $\tau_{r r}^{\mathrm{M}}\left(R_{2}, t\right)$ are the stresses at the inner and outer interfaces, respectively, and $\tau_{r r}^{\mathrm{L}}\left(R_{2}, t\right)$ is the stress in the liquid at the outer interface (CHURCH, 1995).

The boundary conditions from conservation of radial momentum can be stated as

$$
p_{\mathrm{g}}\left(R_{3}, t\right)+\tau_{r r}^{\mathrm{M}}\left(R_{3}, t\right)=P_{\mathrm{M}}\left(R_{3}, t\right)+\frac{2 \sigma_{3}}{R_{3}}
$$

and

$$
\begin{aligned}
P_{\mathrm{M}}\left(R_{4}, t\right)-\tau_{r r}^{\mathrm{M}}\left(R_{4}, t\right)= & P_{\mathrm{L}}\left(R_{4}, t\right)-\tau_{r r}^{\mathrm{L}}\left(R_{4}, t\right) \\
& +\frac{2 \sigma_{4}}{R_{4}}+P(t),
\end{aligned}
$$

where $P_{\mathrm{g}}\left(R_{1}, t\right)$ is the instantaneous pressure inside the void, and $\sigma_{3}$ and $\sigma_{4}$ are the surface tensions at the two respective interfaces of the outer membrane. Combining (11) with the boundary conditions in (12) and (13) yields

$$
\begin{aligned}
\rho_{\mathrm{L}} R_{3} \ddot{R}_{3}\left[1+\left(\frac{\rho_{\mathrm{L}}-\rho_{\mathrm{M}}}{\rho_{\mathrm{M}}}\right) \frac{R_{3}}{R_{4}}\right] \\
+\rho_{\mathrm{L}} \dot{R}_{3}^{2}\left[\frac{3}{2}+\left(\frac{\rho_{\mathrm{L}}-\rho_{\mathrm{M}}}{\rho_{\mathrm{M}}}\right)\left(\frac{4 R_{4}^{3}-R_{3}{ }^{3}}{R_{4}{ }^{3}}\right)\right] \\
=p_{\mathrm{g}}\left(R_{3}, t\right)-\frac{2 \sigma_{3}}{R_{3}}-\frac{2 \sigma_{4}}{R_{4}}-p_{0}-P(t) \\
+3 \int_{R_{3}}^{R_{4}} \frac{\tau_{r r}^{\mathrm{M}}}{r} \mathrm{~d} r+3 \int_{R_{4}}^{\infty} \frac{\tau_{r r}^{\mathrm{L}}}{r} \mathrm{~d} r
\end{aligned}
$$


It now remains to determine the two integrals in (14). Where the first integral describes the rheological properties of the membrane, and the latter integral describes the damping from the surrounding fluid.

Assuming the void is surrounded by a Newtonian viscous liquid, the shear viscous stress can be expressed as

$$
\tau_{r r}^{\mathrm{L}}=2 \eta_{\mathrm{L}} \frac{\partial v}{\partial r}
$$

The last integral in (14) can now be determined using (6), yielding an expression for the effect of a viscous surrounding liquid:

$$
3 \int_{R_{2}}^{\infty} \frac{\tau_{r r}^{\mathrm{L}}}{r} \mathrm{~d} r=-4 \eta_{\mathrm{L}} \frac{R_{3}{ }^{2}}{R_{4}{ }^{3}} \dot{R}_{3} .
$$

The general Rayleigh-Plesset-like equation for a cell with a finite membrane and surrounded by a Newtonian viscous liquid is obtained by substituting

$$
p_{\mathrm{g}}=p_{\mathrm{g} 0}\left(\frac{R_{30}{ }^{3}-R_{2}{ }^{2}}{{R_{3}{ }^{3}-R_{2}{ }^{3}}^{\gamma}}\right)^{\gamma}
$$

for $p_{\mathrm{g}}\left(R_{1}, t\right)$ and (16) for the last integral in (14):

$$
\begin{aligned}
\rho_{\mathrm{L}} R_{3} \ddot{R}_{3} & {\left[1+\left(\frac{\rho_{\mathrm{L}}-\rho_{\mathrm{M}}}{\rho_{\mathrm{M}}}\right) \frac{R_{3}}{R_{4}}\right] } \\
+ & \rho_{\mathrm{L}} \dot{R}_{3}^{2}\left[\frac{3}{2}+\left(\frac{\rho_{\mathrm{L}}-\rho_{\mathrm{M}}}{\rho_{\mathrm{M}}}\right)\left(\frac{4 R_{4}{ }^{3}-R_{3}{ }^{3}}{R_{4}{ }^{3}}\right)\right] \\
= & p_{\mathrm{g} 0}\left(\frac{R_{30}{ }^{3}-R_{2}{ }^{2}}{R_{3}{ }^{3}-R_{2}{ }^{3}}\right)^{\gamma}-\frac{2 \sigma_{3}}{R_{3}}-\frac{2 \sigma_{4}}{R_{4}}-p_{0}-P(t) \\
& -4 \eta_{\mathrm{L}} \frac{R_{3}{ }^{2}}{R_{4}{ }^{3}} \dot{R}_{3}+3 \int_{R_{3}}^{R_{4}} \frac{\tau_{r r}^{\mathrm{M}}}{r} \mathrm{~d} r .
\end{aligned}
$$

\subsection{Newtonian viscous liquid outer cell membrane}

Now let us assume that the outer monolayer membrane of a cell can be regarded as a Newtonian viscous liquid, where the viscous stress in the membrane $\tau_{r r}^{\mathrm{M}}$ is related to the membrane viscosity $\eta_{\mathrm{M}}$ by (DoINIKOV, DAYTON, 2007):

$$
\tau_{r r}^{\mathrm{M}}=2 \eta_{\mathrm{M}} \frac{\partial v}{\partial r}
$$

The remaining integral in (18) describes the shear viscosity in the Newtonian liquid membrane, which dampens the radial response from the void as it is excited by an acoustic pulse. Substituting (19) and using both (7) and (8), the integral can be written in terms of radial displacement:

$$
3 \int_{R_{3}}^{R_{4}} \frac{\tau_{r r}^{\mathrm{M}}}{r} \mathrm{~d} r=-4 \eta_{\mathrm{M}} \frac{R_{4}{ }^{3}-R_{3}{ }^{3}}{R_{3} R_{4}{ }^{3}} \dot{R}_{3} .
$$

Substituting (20) into (18) gives a Rayleigh-Plesset-like equation for a cell with a Newtonian membrane of finite thickness surrounded by a Newtonian viscous liquid:

$$
\begin{aligned}
\rho_{\mathrm{L}} R_{3} & \ddot{R}_{3}\left[1+\left(\frac{\rho_{\mathrm{L}}-\rho_{\mathrm{M}}}{\rho_{\mathrm{M}}}\right) \frac{R_{3}}{R_{4}}\right] \\
& +\rho_{\mathrm{L}} \dot{R}_{3}^{2}\left[\frac{3}{2}+\left(\frac{\rho_{\mathrm{L}}-\rho_{\mathrm{M}}}{\rho_{\mathrm{M}}}\right)\left(\frac{4 R_{4}{ }^{3}-R_{3}{ }^{3}}{R_{4}{ }^{3}}\right)\right] \\
= & p_{\mathrm{g} 0}\left(\frac{R_{30}{ }^{3}-R_{2}{ }^{2}}{R_{3}{ }^{3}-R_{2}{ }^{3}}\right)^{\gamma}-\frac{2 \sigma_{3}}{R_{3}}-\frac{2 \sigma_{4}}{R_{4}}-p_{0}-P(t) \\
& -4 \eta_{\mathrm{L}} \frac{R_{3}{ }^{2}{ }_{R_{4}}{ }^{3}}{R_{3}-4 \eta_{\mathrm{M}}} \frac{R_{4}{ }^{3}-R_{3}{ }^{3}}{R_{3} R_{4}{ }^{3}} \dot{R}_{3} .
\end{aligned}
$$

\section{Solution}

To simulate a spherically oscillating cell, parameters measured on living blood cells were used. Bilayer cell membranes are $20 \mathrm{~nm}$ in thickness, whereas the void separating the individual monolayers is only $2 \mathrm{~nm}$ in thickness (BOAL, 2012). The shear viscosity of red blood cell membranes is in the order of $10^{3} \mathrm{~Pa} \cdot \mathrm{s}$ (TRAN-SON-TAY et al., 1984). The sizes of cells greatly vary. Red blood cells have in axial view an outer radius of $3 \mu \mathrm{m}$.

Equation (21) was solved numerically using the ode45 algorithm of MATLAB ${ }^{\circledR}$ (The MathWorks, Inc., Natick, MA, USA). The parameters chosen were: $\rho_{\mathrm{M}} \approx$ $\rho_{\mathrm{L}}=10^{3} \mathrm{~kg} \cdot \mathrm{m}^{-3}, R_{40}=3 \mu \mathrm{m},\left(R_{40}-R_{30}\right)=9 \mathrm{~nm}$, $\left(R_{30}-R_{2}\right)=2 \mathrm{~nm}, \rho_{\mathrm{g} 0}=1 \mathrm{~kg} \cdot \mathrm{m}^{-3}, \gamma=1.4, \sigma_{3}=$ $\sigma_{4}=0.025 \mathrm{~N} \cdot \mathrm{m}^{-1}, p_{0}=10^{5} \mathrm{~Pa}, \eta_{\mathrm{L}}=10^{-3} \mathrm{~Pa} \cdot \mathrm{s}$, and $\eta_{\mathrm{M}}=10^{3} \mathrm{~Pa} \cdot \mathrm{s}$.

The driving pulse consisted of a 10-cycles sinusoid wave, with a centre frequency of $1 \mathrm{MHz}$ or $3 \mathrm{MHz}$. The number of cycles chosen is typical for the upper pulse duration in commercial ultrasound equipment, whereas the centre frequencies are typical for those used in clinical cardiac and gastroenterologic ultrasonic imaging. Acoustic amplitudes ranged from $300 \mathrm{kPa}$ to $20 \mathrm{MPa}$. The low acoustic pressures were chosen to simulate clinically safe ultrasound, the higher pressures to investigate under what conditions ultrasound-induced cell damage should be permanent.

\section{Results and discussion}

Figures 2-4 show expansion-time curves of red blood cells under sonication. In all these figures, it can be observed, that all oscillations of the void are highly asymmetric, i.e., greater expansive excursions than contractive excursions. This is a direct result of the incompressibility of the cellular content and of the nonlinearity of (21). It may be interesting to mention, that if the density of the outer membrane were different from the density of the surrounding fluid, more asymmetry should have occurred: According to (18), 


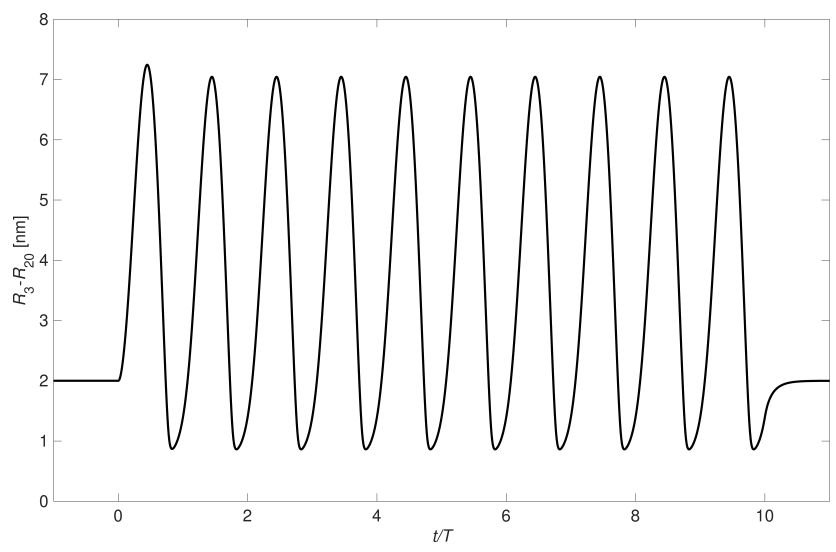

Fig. 2. Expansion-time curve of a red blood cell under $1-\mathrm{MHz}$ sonication. The acoustic amplitude is $300 \mathrm{kPa}$. Time $t$ has been normalised by period $T$.

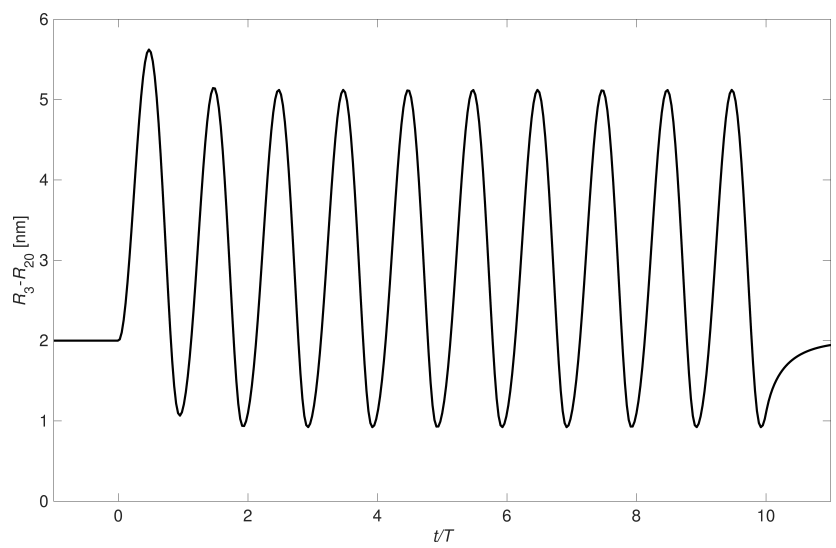

Fig. 3. Expansion-time curve of a red blood cell under 3 -MHz sonication. The acoustic amplitude is $500 \mathrm{kPa}$. Time $t$ has been normalised by period $T$.

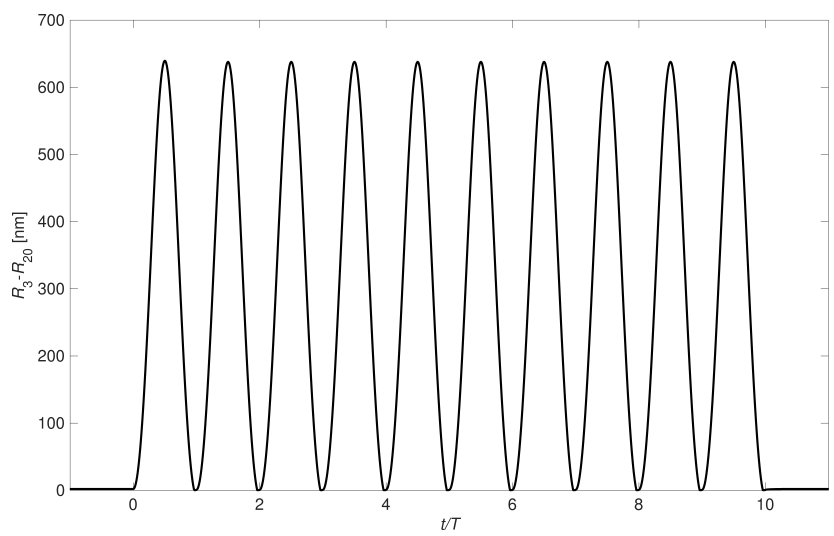

Fig. 4. Expansion-time curve of a red blood cell under $1-\mathrm{MHz}$ sonication. The acoustic amplitude is $20 \mathrm{MPa}$. Time $t$ has been normalised by period $T$.

it follows from the first term on the left-hand side that the acceleration increases if $\rho_{\mathrm{L}}>\rho_{\mathrm{M}}$, and the acceleration decreases if $\rho_{\mathrm{L}}<\rho_{\mathrm{M}}$. The ratios of the densities effects the second term on the left-hand side in a similar way, decreasing and increasing the degree of nonlinearity. The first term on the right-hand side is a different form of a Rayleigh-Plesset-like equation, describing the radial pulsation of a gas bubble. The pressure inside a cell is larger than in a gas bubble under the same conditions.

Figures 2 and 3 both demonstrate a maximum void expansion of more than $5 \mathrm{~nm}$, i.e., more than $2.5 \times$ the initial void thickness, despite the high viscosity $\left(\eta_{\mathrm{M}}=10^{3} \mathrm{~Pa} \cdot \mathrm{s}\right)$ of the outer monolayer membrane. The acoustic amplitudes in both computations were chosen to correspond to a mechanical index of 0.3 (APFel, Holland, 1991): At a transmit frequency of $1 \mathrm{MHz}$, acoustic amplitudes of pulsed ultrasound below $300 \mathrm{kPa}$ are considered safe in neonatal scans, whereas at a transmit frequency of $3 \mathrm{MHz}$, acoustic amplitudes of pulsed ultrasound below $500 \mathrm{kPa}$ are considered safe in neonatal scans (Postema, 2011).

Hence, even at low acoustics amplitudes, the void inside the bilayer membrane oscillates to multiple times its initial thickness. These excursions are not enough to permanently damage a red blood cell.

Recently, Li et al. (2013) measured that red blood cells need to be stretched to an area corresponding to $20 \%$ increase of their resting radius. Figure 4 shows that such excursions can be achieved during sonication at $1 \mathrm{MHz}$ with an acoustic pressure amplitude of $20 \mathrm{MPa}$. This pressure amplitude corresponds to $10 \times$ the threshold of inertial cavitation (APFEL, Holland, 1991). Hence, permanent cell damage caused by the oscillating bilayer void can be neglected in clinical situations. However, diffusive processes into the void have not been accounted for in our model. Such processes might inflate the void over multiple cycles.

The purpose of this paper was to propose a model with less unknown parameters than the sonophore. Introducing compressible membranes, bulk viscosity and thermal conductivity would, albeit representing a more realistic situation, complicate the model with unknown material properties. However, real excursion amplitudes should be even lower due to these additional damping terms.

It can be noted that, given the incompressibility of the inner monolayer, the radial oscillations of a cell are governed by the same set of equations as those of a forced antibubble (Koтopoulis et al., 2015).

\section{Conclusions}

We derived the spatio-temporal cell dynamics for a spherically symmetric single cell, whilst regarding the cell bilayer membrane as two monolayer Newtonian viscous liquids, separated by a thin gas void.

Given the incompressibility of the inner monolayer, the radial oscillations of a cell are governed by the same set of equations as those of a forced antibubble. Evidently, these equations must hold for liposomes under sonication, as well. 


\section{References}

1. Apfel R.E., Holland C.K. (1991), Gauging the likelihood of cavitation from short-pulse, low-duty cycle diagnostic ultrasound, Ultrasound in Medicine \& Biology, 17, 2, 179-185.

2. Bao S., Thrall B.D., Miller D.L. (1997), Transfection of a reporter plasmid into cultured cells by sonoporation in vitro, Ultrasound in Medicine \& Biology, 23, 6, 953-959.

3. BoAl D. (2012), Mechanics of the Cell, University Press, Cambridge.

4. Church C.C. (1995), The effects of an elastic solid surface layer on the radial pulsations of gas bubbles, Journal of the Acoustical Society of America, 97, 3, $1510-1521$.

5. Delalande A., Kotopoulis S., Rovers T., PiChon C., Postema M. (2011), Sonoporation at a low mechanical index, Bubble Science, Engineering and Technology, 3, 1, 3-11.

6. Delalande A., Postema M., Mignet N., MiDOUX P., PiCHON C. (2012), Ultrasound and microbubble-assisted gene delivery: recent advances and ongoing challenges, Therapeutic Delivery, 3, 10, 11991215.

7. Doinikov A.A., Dayton P.A. (2007), Maxwell rheological model for lipid-shelled ultrasound microbubble contrast agents, Journal of the Acoustical Society of America, 121, 6, 3331-3340.

8. IsEnBerg C. (1992), The science of soap films and soap bubbles, Dover edition, General Publishing Company, Don Mills.

9. Kotopoulis S., Johansen K., Gilja O.H., Poortinga A.T., Postema M. (2015), Acoustically active antibubbles, Acta Physica Polonica A, 127, 1, 99-102.

10. Krasovitski B., Frenkel V. Shoham S., KimMEL E. (2011), Intramembrane cavitation as a unifying mechanism for ultrasound-induced bioeffects, Procee- dings of the National Academy of Sciences, 108, 8, $3258-3263$.

11. Kudo N., Okada K., Yamamoto K. (2009), Sonoporation by single-shot pulsed ultrasound with microbubbles adjacent to cells, Biophysical Journal, 96, 12, 4866-4876.

12. Landau L., Lifshitz E. (1986), Theory of Elasticity, Butterworth-Heinemann, Oxford.

13. Leighton T.G. (1994), The Acoustic Bubble, Academic Press, London.

14. Li F., Chan C.U., Ohl C.D. (2013), Yield strength of human erythrocyte membranes to impulsive stretching, Biophysical Journal, 105, 4, 872-879.

15. Mazzawi N., Postema M., Kimmel E. (2015), Bubble-like response of living blood cells and microparticles in an ultrasound field, Acta Physica Polonica A, 127, 1, 103-105.

16. Postema M. (2011), Fundamentals of Medical Ultrasonics, Spon Press, London.

17. Prentice P., Cuschieri A., Dholakia K., PrausNitz M., CAmpbell P. (2005), Membrane disruption by optically controlled microbubble cavitation, Nature Physics, 1, 107-110.

18. Tran-Son-Tay R., Sutera S.P., Rao P.R. (1984), Determination of red blood cell membrane viscosity from rheoscopic observations of tank-treading motion, Biophysical Journal, 46, 1, 65-72.

19. van Wamel A., Kooiman K., Harteveld M., Emmer M., ten Cate F.J., Versluis M., De Jong N. (2006), Vibrating microbubbles poking individual cells: drug transfer into cells via sonoporation, Journal of Controlled Release, 112, 2, 149-155.

20. Walther T., Postema M. (2016), Device for the identification, separation and/or cell type-specific manipulation of at least one cell of a cellular system, United States Patent Application US 2016/0060615 A1. 